\title{
In vitro Induction of Banana Autotetraploids by Colchicine Treatment of Micropropagated Diploids
}

\author{
S.D. Hamill A, M.K. Smith A and W.A. Dodd ${ }^{\mathrm{B}}$ \\ ${ }^{A}$ Queensland Department of Primary Industries, Maroochy Horticultural Research Station, \\ PO Box 5083, SCMC, Nambour, Qld 4560, Australia. \\ ${ }^{B}$ Queensland University of Technology, School of Life Sciences, \\ PO Box 2334, Brisbane, Qld 4000, Australia.
}

\begin{abstract}
Alternative breeding strategies, based on colchicine-induced autotetraploids, have been proposed as a means of introducing disease resistance into banana breeding programs. This paper describes techniques for the in vitro induction of banana autotetraploids by the use of colchicine on cultured explants. The technique can be readily applied and large numbers of autotetraploids produced. The optimum treatment involved immersing shoot tips in a $0.5 \% \mathrm{w} / \mathrm{v}$ colchicine solution for $2 \mathrm{~h}$ under aseptic conditions. Dimethyl sulfoxide (DMSO) was applied with the colchicine treatments to increase cell permeability and so absorption of colchicine, resulting in the optimum treatment unchanged at $0.5 \%$ colchicine, but including the addition of $2 \% \mathrm{v} / \mathrm{v}$ DMSO. Of the shoot tips treated over $30 \%$ were induced to the autotetraploid level.

Methods for in vitro selection of induced tetraploids from treated diploid plantlets were also developed. Tetraploid plants were more robust with thicker pseudostems, roots and broader leaves than diploids and they could be selected on these morphological characteristics. Mean stomatal lengths of diploid banana plants growing in vitro were significantly smaller $(16.0 \mu \mathrm{m})$ than the tetraploids $(26.9$ $\mu \mathrm{m}$ ) and were used as a more reliable indicator of ploidy than morphological criteria alone. A root tip squash technique using carbol fuchsin was developed for positive confirmation of ploidy change by chromosome counts. Although chimerism and reversion to the diploid form occurred, it was not considered a problem because of the large number of autotetraploids induced. Stable autotetraploids were recovered and established in the field and were characterised by their large, drooping leaves and thick pseudostems. They have retained these characteristics for more than 3 years in the field.
\end{abstract}

\section{Introduction}

Bananas are one of the most important fruits grown throughout the world both as a subsistence and export crop (Simmonds 1966). Cavendish (AAA) clones are the dessert bananas primarily used for export and these triploid Musa acuminata types have been the mainstay of the industry for the past 30-40 years. There are now major threats facing the world's Cavendish-based industries, notably Fusarium wilt (Fusarium oxysporum f.sp. cubense race 4) and black Sigatoka (Mycosphaerella fijiensis var. difformis), and resistance to these two major diseases is the principal aim of breeding for banana improvement (Persley and De Langhe 1987). The strain of Fusarium wilt that attacks Cavendish, race 4, is of particular concern to the Australian industry as there are no effective chemical means of control and no resistant cultivars that can replace Cavendish.

Breeding bananas poses problems to the plant breeder as most of the commercially acceptable bananas are parthenocarpic triploids which produce few if any seeds, and seedlessness of the fruit is a characteristic which must be retained by the breeder. Conventional banana breeding programs have relied on 'Gros Michel' (AAA), and its shorter somatic mutants, as the female parent because they occasionally set seed when hand pollinated (Rowe and Richardson 1975; Rowe 1984). Cavendish are unsuitable because they 
are entirely sterile (Shepherd 1987). The unreduced set of gametes produced by Gros Michel when fertilised by pollen recovered from diploid males produce tetraploid progeny that retain many of the characteristics of the female parent. Unfortunately, Gros Michel is highly susceptible to both race 1 and race 4 Fusarium wilt (Pegg and Langdon 1987) and tetraploid progeny retain susceptibility to Fusarium wilt.

Alternative breeding strategies based on colchicine-induced autotetraploids have been proposed as a means of introducing disease resistance into breeding programs (Vakili 1967; Stover and Buddenhagen 1986). By using improved, disease-resistant diploid bananas, and inducing autotetraploids from these, greater genetic diversity can be introduced into a breeding population. However, tetraploid banana plants in themselves are usually inferior to triploid clones. Their main disadvantages are large, drooping leaves and a weak petiole, which often results in a high incidence of wind damage to the plants (Stover and Simmonds 1987). Their importance will be in breeding programs where the best tetraploid clones can be crossed with superior diploids to yield horticulturally desirable triploid progeny. Because these triploids will be produced from a wider genetic base (not limited by Gros Michel lineage), the chances of obtaining a superior cultivar are increased.

Although methods for the colchicine induction of polyploids are common for a range of plant species (Derman 1940; Krythe and Wellensieke 1952; Burnham 1962; Sanford 1983), few relate to the in vitro application of colchicine and there are no published reports on the in vitro induction of polyploids in banana. A technique is described here for the in vitro induction of banana autotetraploids by the use of colchicine. Methods for the early detection of autotetraploids are also described, being based on morphological differences and changes in stomatal size, and later confirmed by counting chromosomes in root-tip squashes. An improved diploid (AA) Musa acuminata type banana, 'SH - 3362', obtained from Dr P. Rowe, Fundacion Hondurena de Investigacion Agricola (FHIA), has a high level of resistance to race 4 Fusarium wilt in Australia (Pegg and Langdon 1987; Brake et al. 1990) and was therefore selected for use in this study.

\section{Materials and Methods}

\section{Plant Material}

Three banana clones were used:

(1) 'SH-3362', a diploid Musa acuminata (AA) clone that has shown a high level of resistance to race 4 of Fusarium oxysporum f. sp. cubense in Australia and reportedly has resistance to black Sigatoka,

(2) 'SH-3436', a tetraploid Musa acuminata (AAAA) clone ('SH-3362' was in the pedigree), and

(3) 'Williams' a triploid Musa acuminata (AAA) clone of the Cavendish subgroup.

\section{Culture Conditions}

Banana material was micropropagated using the method described by Smith and Drew (1990). Tissue cultures were initiated from shoot tips taken from banana suckers and placed onto MS medium (Murashige and Skoog 1962) containing $2.5 \mathrm{mg} \mathrm{L}^{-1}$ benzylaminopurine (BAP), $20 \mathrm{~g} \mathrm{~L}^{-1}$ sucrose, 100 mg L $\mathrm{L}^{-1}$ myo-inositol, solidified with $8 \mathrm{~g} \mathrm{~L}^{-1}$ Difco-Bacto agar, and the $\mathrm{pH}$ was adjusted to 6.0 . Multiplication occurred by subdividing shoot or bud clusters and reculturing these individual pieces on fresh medium every 4-6 weeks. Material was grown in $125 \mathrm{~mL}$ borosilicate glass pomade bottles before being used in various experiments. The cultures were incubated at $28^{\circ} \mathrm{C}$ under cool white fluorescent tubes which provided a photon flux density at the culture surface of $60 \mu \mathrm{mol} \mathrm{m} \mathrm{m}^{-2} \mathrm{~s}^{-1}$ for a 16 $\mathrm{h}$ photoperiod. Explants, with the roots trimmed and ensheathing leaves removed $4-5 \mathrm{~mm}$ above the shoot tip, were used exclusively throughout the colchicine experiments.

Prior to field establishment, explants were transferred to hormone-free MS media for root initiation and shoot elongation. When individual shoots produced expanded leaves and roots they were transferred to pasteurised potting mix composed of sand and peat and deflasked and hardened off using the method described by Daniells and Smith (1991). 
Morphological Comparison of Diploid and Tetraploid Bananas Grown in vitro

\section{Gross morphology}

The tetraploid, SH-3436, and a diploid parent, SH-3362, were compared in vitro to develop selection criteria enabling the early detection of tetraploids following colchicine treatment. Ten explants of each clone were placed into a sterile plastic tub, $110 \mathrm{~mm}$ diam. $\times 80 \mathrm{~mm}$, containing hormone-free MS media. Plants were evenly spaced and eight tubs of each clone were prepared and arranged randomly on a shelf. Commencing at week 2, a tub from each clone was used for destructive measurements of each plantlet and measurements were taken weekly over an 8 week period. Measurements included: (1) height, measured from the base of the plant to the base of the newly emerging leaf; (2) pseudostem diameter, measured $10 \mathrm{~mm}$ from the base below the oldest leaf; (3) root length; (4) root diameter, measured midway along the length of the root; (5) leaf length, breadth and petiole length, measured as the leaf emerged and as it expanded after 1 or 2 weeks; (6) leaf index, measured as length divided by breadth.

\section{Stomatal characters}

The diploid clone SH-3362 and tetraploid SH-3436 also had stomatal measurements taken over the 8 week period. Stomata from Williams (AAA, Cavendish subgroup), as well as tetraploids and octaploids produced from the colchicine treatments, were also examined as described below.

Stomata were measured from an imprint of the leaf surface obtained by painting clear finger-nail polish on the leaf, allowing it to dry and peeling it off. For stomatal length, 10 stomata were measured from upper and lower surfaces of the middle of each leaf at $400 \mathrm{x}$ magnification, and stomatal density was recorded at $200 \mathrm{x}$ magnification as number in the field of view $\left(1.0 \mathrm{~mm}^{2}\right)$ on both leaf surfaces.

\section{Colchicine Treatments}

Only the diploid clone SH-3362 was treated with colchicine. Colchicine is readily dissolved in aqueous solution and is unaffected by autoclaving (Blakeslee and Avery 1937). Therefore colchicine at concentrations of $0,0.05,0.25,0.5,0.75$, and $1.0 \% \mathrm{w} / \mathrm{v}$ was added directly to $125 \mathrm{~mL}$ Erlenmeyer flasks containing $25 \mathrm{~mL}$ of MS medium. The Erlenmeyer flasks were autoclaved at $121^{\circ} \mathrm{C}$ for $20 \mathrm{~min}$. Explants were placed onto hormone-free MS media until they were showing signs of active growth ( 8 days), then placed in $25 \mathrm{~mL}$ liquid MS medium in $125 \mathrm{~mL}$ Erlenmeyer flasks on an orbital shaker at $100 \mathrm{rpm}$ and shaken for $24 \mathrm{~h}$ under conditions described previously. The explants were then transferred to colchicine treatments at the commencement of the light cycle as cell division may be synchronised by a light stimulus and more cells become active after exposure to light following a dark period (Iyer and Randhawar 1965). The explants were immersed in the various colchicine treatments for $0.5,2$ or $4 \mathrm{~h}$. Explants were then removed from these treatments and washed thoroughly on a sieve with $500 \mathrm{~mL}$ of sterile distilled water and then placed onto solid MS medium. Fifty explants were used for each treatment and the experiment was repeated. Explants were examined for ploidy changes three subcultures after treatment. Suspected autotetraploids were selected using the morphological criteria developed for in vitro grown diploid and tetraploid plantlets. Putative tetraploids were grouped and stomata were measured to more specifically determine ploidy. Finally the chromosome number was determined from a root-tip squash. Root tips were fixed in Farmers fixative (glacial acetic acid : 95\% ethanol, $1: 2$ ) for a minimum of $72 \mathrm{~h}$. Roots were rinsed in water and softened in $5 \mathrm{~N} \mathrm{HCl}$ for $25 \mathrm{~min}$ at room temperature then squashed and stained with an altered form of carbol fuchsin (Martens and Reisch 1988).

After determining that the optimum colchicine treatment for inducing tetraploids from single shoot tips of diploids was $0.5 \%$ for $2 \mathrm{~h}$, fresh multiplying shoot tips were exposed to $0.5 \%$ colchicine for 2 or $4 \mathrm{~h}$. MS medium, containing $2.5 \mathrm{mg} \mathrm{L}^{-1} \mathrm{BAP}$, was used to induce axillary budding, and explants were subcultured three times on this media prior to colchicine treatment. Fifty plants were used for each treatment and the experiment was repeated. The treated explants were then placed onto solid MS media containing $2.5 \mathrm{mg} \mathrm{L}^{-1} \mathrm{BAP}$ and subcultured for a further three cycles. During this period actively growing axillary and adventitious buds were produced. Explants were then placed on hormone-free MS media to induce formation of elongated shoots and roots for determination of ploidy as above.

\section{Dimethyl Sulfoxide (DMSO) with Colchicine Treatments}

Because DMSO increases cell permeability and allows increased absorption of chemicals, the treatment times and colchicine concentrations determined previously were reduced for experiments 
incorporating DMSO. DMSO at 0,2 or $4 \% \mathrm{v} / \mathrm{v}$ was incorporated with colchicine concentrations at $0.25,0.4$ or $0.5 \% \mathrm{w} / \mathrm{v}$ for $2 \mathrm{~h}$. Explants were treated and examined as described previously. Fifty explants were used for each treatment and the experiment was repeated.

\section{Statistical Analysis}

Percentage data were arcsine-transformed for statistical calculations. All data were analysed using analysis of variance.

\section{Results}

\section{Morphological Differences between Diploid and Tetraploid Bananas Grown in vitro}

At the end of the 8 week culture cycle, the diploid and tetraploid plants could be distinguished on morphological features alone. The tetraploids had significantly thicker pseudostems $(P<0.001)$ with larger leaves and roots, and were generally more robust (Fig. $1 a$ ). There were no differences in height or petiole length between the diploid and tetraploid plants. From week 5 onward roots were significantly thicker $(P<0.001)$ (Fig. $1 b)$ and emerging leaves were significantly broader $(P<0.001)$ (Fig. $1 c)$ on the tetraploid plant. Leaf length did not alter between the tetraploid and diploid, but the leaf of the tetraploid was rounder and the leaf index was lower $(P<0.001)$ on the tetraploid due to increased breadth.

a
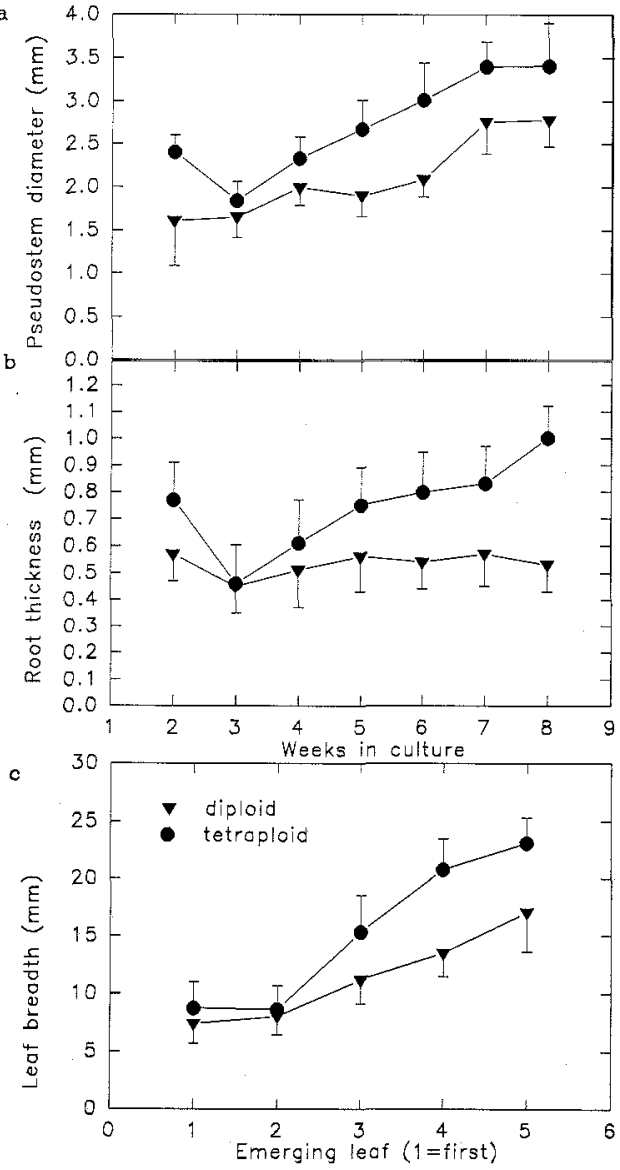

Fig. 1. Morphological measurements taken either over time $(a$ and $b)$ or as leaves emerged $(c)$ from diploid and tetraploid banana plants grown in vitro. Shoot tip explants were cultured in Murashige and Skoog media without phytohormones, for 8 weeks. Vertical bars represent standard error from 10 replicates. (a) Pseudostem diameter measured in vitro. (b) Root thickness measured in vitro. (c) Leaf breadth measured in vitro. 
Stomatal size and density differed significantly between the micropropagated diploid and tetraploid bananas. The tetraploid had larger stomata in the range of $23.5-32.75 \mu \mathrm{m}$ with a mean of $26.9 \mu \mathrm{m}$ while diploid stomatal length ranged from 12.25 to $20.75 \mu \mathrm{m}$ with a mean of $16.0 \mu \mathrm{m}(P<0.001)$. There was no overlap in ranges of stomatal length.

There was no variation in stomatal length between the upper or lower leaf surfaces nor was there variation between plants from different culture bottles. The diploid had significantly more stomata than the tetraploid $(P \leq 0.001)$ and, as expected, the upper leaf surface had fewer stomata than the lower leaf surface.

Stomatal lengths from the triploid, $c v$.Williams, ranged from 17.5 to $33.25 \mu \mathrm{m}$ with a mean of $25.9 \mu \mathrm{m}$. This range fell between the diploid and tetraploid ranges and overlapped them. Stomata from octaploid bananas produced from the colchicine treatments were also examined and found to be larger than the tetraploid, with a range of $32.1-37.4 \mu \mathrm{m}$ and a mean of $35.01 \mu \mathrm{m}$. The mean stomatal lengths of the diploid, triploid, tetraploid and octaploid were significantly different from each other $(P<0.001)$ and increased as ploidy increased: The relationship can be described by a curvilinear regression: $y=1.32893+0.78814 x+-0.000417 x^{2}$, $\left(R^{2}=0.87954\right)$. The mean stomatal length of the field-grown banana plants was $22.1 \mu \mathrm{m}$ for the diploid, $32.2 \mu \mathrm{m}$ for the triploid and $34.3 \mu \mathrm{m}$ for the tetraploid and, like those cultured in vitro, were significantly different from each other $(P<0.001)$. The field-grown plants had significantly larger stomata than the micropropagated plants $(P<0.001)$; however, the trend of increasing stomatal size with increasing ploidy was consistent.

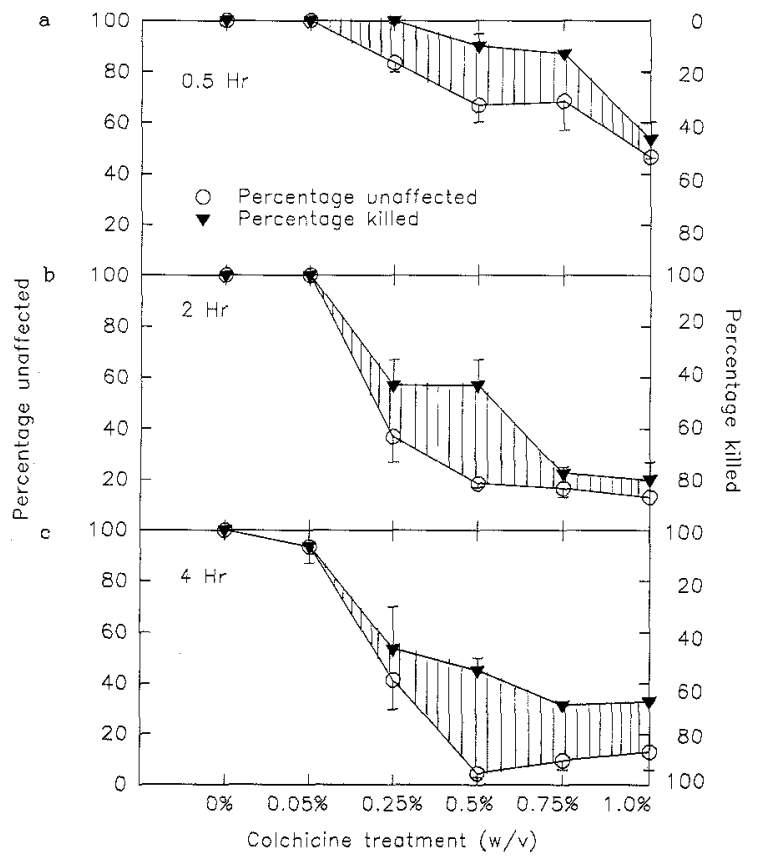

Fig. 2. Percentage of polyploid plants induced following exposure of diploid explants to colchicine. Polyploid plants include tetraploids, octaploids and aneuploids. Banana shoot tip explants were exposed to colchicine dissolved in liquid MS medium in vitro. Vertical bars represent standard error. Percentage unaffected $(0$, left $Y$ axis), percentage killed ( $\nabla$, right $Y$ axis). The shaded area indicates the percentage of explants induced to a polyploid level.

(a) Colchicine applied for $0.5 \mathrm{~h}$

(b) Colchicine applied for $2 \mathrm{~h}$

(c) Colchicine applied for $4 \mathrm{~h}$.

\section{Colchicine Treatments}

Explants treated with colchicine were initially slower growing than untreated control plants and mortality increased from 0 to over $70 \%$ in some treatments (Fig. 2). Often explants induced to a higher ploidy had leaves that at first emerged with rosette-like growth. Some of the octaploid and aneuploid plants were stunted, slow growing with thick leaves that were sometimes chimeric, and often had sparse root systems with stunted thick discoloured roots. 
Many of the treated diploids were unaffected by the colchicine treatment. They grew most rapidly in the first subculture cycle and had pale, curled leaves which are typical of micropropagated SH-3362. By contrast, autotetraploid plants were darker green with broad flat leaves and thicker pseudostems. These morphological features were a useful means of selecting putative tetraploids. Colchicine began to elicit a response at $0.25 \% \mathrm{w} / \mathrm{v}$ colchicine, or at higher concentrations (Fig. 2). Treatments for 2 or $4 \mathrm{~h}$ resulted in larger numbers of plants with higher ploidy levels $(P<0.001)$ and a higher death rate than the $0.5 \mathrm{~h}$ treatment. The $0.5 \% \mathrm{w} / \mathrm{v}$ colchicine treatment was significantly better for inducing tetraploids than any other treatment $(P<0.001)$ (Fig. 3). The optimal treatment for the induction of autotetraploids was $0.5 \%$ colchicine for $2 \mathrm{~h}$. In this treatment, $31.5 \%$ of the total explants treated were autotetraploids. More aneuploids and octaploids were induced by the 4-h colchicine treatments $(P \leq 0.05)$, than with 0.5 and $2 \mathrm{~h}$ treatments. The percentage of explants induced to a higher ploidy was not significantly different after treating either shoot tips (not induced to multiply) or multiplying shoot tips. An average of $52.5 \%$ of survivors were induced to the tetraploid level when multiplying or single shoot tips were exposed to $0.5 \%$ colchicine for 2 or $4 \mathrm{~h}$.

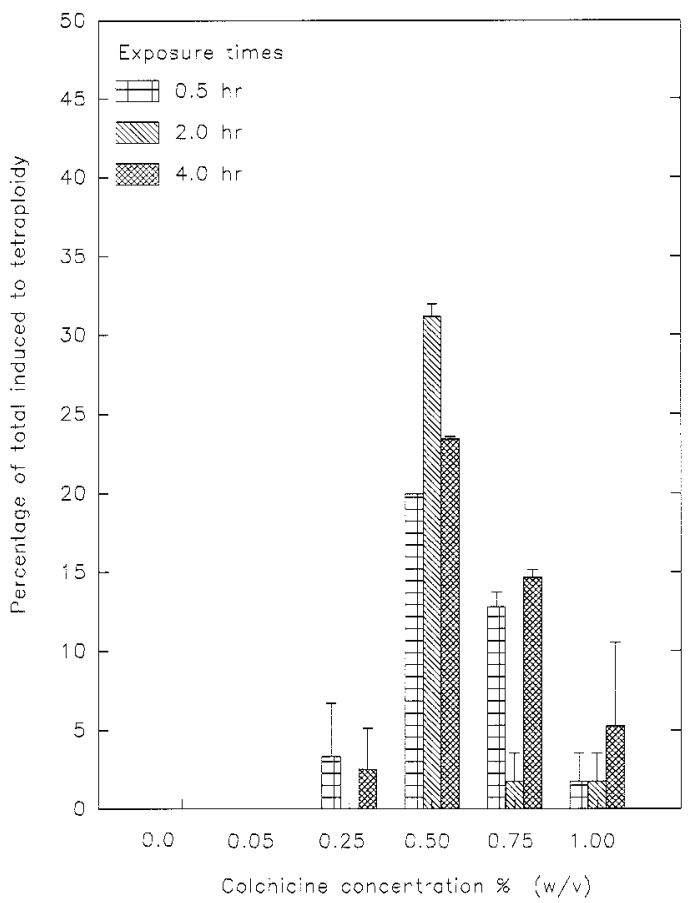

Fig. 3. Percentage of autotetraploid plants induced following exposure of diploid explants to colchicine. Banana shoot tip explants were exposed to colchicine dissolved in liquid MS medium in vitro. Vertical bars represent standard error (where no bars are seen standard error is very low).

\section{Dimethyl Sulfoxide (DMSO) with Colchicine Treatments}

Mortality rate increased when 2 or $4 \%$ DMSO was used in combination with 0.4 and $0.5 \%$ colchicine, compared with 0.4 and $0.5 \%$ colchicine controls $(P \leq 0.001)$ (Fig. $4 a)$, but mortality rate did not differ between DMSO treatments and controls at the $0.25 \%$ colchicine level. The $0.5 \%$ colchicine with 2 or $4 \%$ DMSO produced significantly more tetraploids of the survivors than the other colchicine/DMSO combinations $(P<0.001)$, and $2 \%$ DMSO was better than $4 \%$ DMSO $(P \leq 0.01)$. Colchicine treatments with $2 \%$ DMSO induced a higher percentage of tetraploids from the survivors than from colchicine treatments without DMSO $(P<0.001)$ (Fig. $4 b)$. However, DMSO increased mortality such that the percentage of tetraploids induced from the total treated was not significantly better than colchicine treatment without DMSO (Fig. 4c). 


\section{Stability of Colchicine-treated Plants}

Autotetraploids, and plants of higher ploidy, were planted into the field and some were retained in tissue culture and monitored for reversion and chimerism. Of those lines selected as autotetraploids, $42 \%$ remained stable after 2 years in culture. Of the remaining lines, $15 \%$ were aneuploids, $7 \%$ where chimeric and $36 \%$ reverted to the diploid level. Plants that reverted to diploids, as measured both by stomatal size and chromosome number, either did so within one subculture cycle $(18.6 \%)$, or tissue gradually reverted, as indicated by plants having diploid size stomata and a tetraploid number of chromosomes in root tips as confirmed by chromosome counts $(17.4 \%)$. It was rare to find plants reverting from the tetraploid to diploid level with a diploid number of chromosomes in the root tips while still retaining tetraploid size stomata. Of the octaploid plants, $55.3 \%$ remained stable after 2 years in tissue culture, $14 \%$ reverted to tetraploids and $31 \%$ were aneuploids.

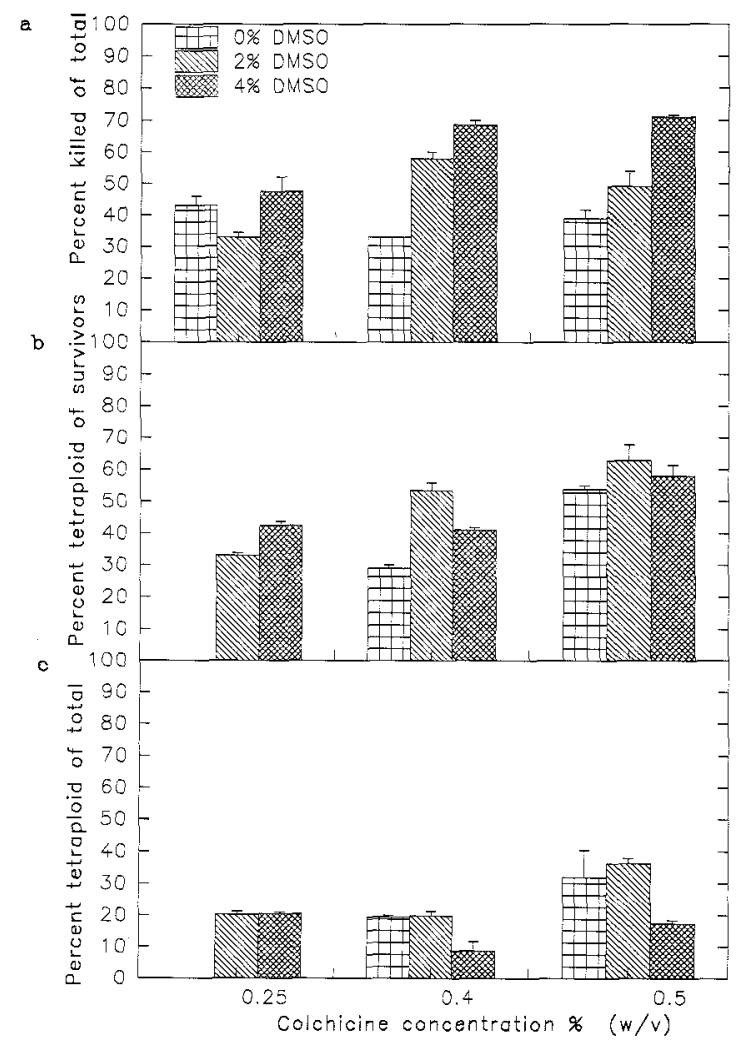

Fig. 4. The effect of colchicine and DMSO treatments on survival of diploid banana plants in vitro. $0.25,0.4$ or $0.5 \%$ w/v colchicine in combination with 0,2 or $4 \% \mathrm{v} / \mathrm{v}$ DMSO were applied for $2 \mathrm{~h}$. Banana shoot tip explants were used for the colchicine/DMSO treatments which was dissolved in liquid MS media before the explants were shaken for $2 \mathrm{~h}$. Vertical bars represent standard error (where there are no error bars standard error is less than 1). (a) Percentage of plants killed from the total treated. (b) Percentage of plants induced to tetraploidy from the plants that survived. (c) Percentage of plants induced to tetraploidy from the total treated.

\section{Discussion}

Obvious morphological differences exist between micropropagated diploid and tetraploid banana plantlets and these criteria were used successfully to select autotetraploids from the colchicine-treated diploids in vitro. Stomatal length was a particularly useful measurement for distinguishing diploids from tetraploids in vitro, and measurements taken from the middle of any surface of any leaf can be used as an indicator of the ploidy of micropropagated banana plantlets. For banana plants grown in vitro or in vivo the higher the chromosome number the larger the stomata. Field-grown clones have significantly larger stomata than those in tissue culture. In field-grown plants, as with tissue cultured ones, stomatal measurements are useful in distinguishing diploids from tetraploids. A proportional relationship between ploidy and stomatal length was also found on field-grown plants by Borges (1971) on a number of clones of various genomic composition. 

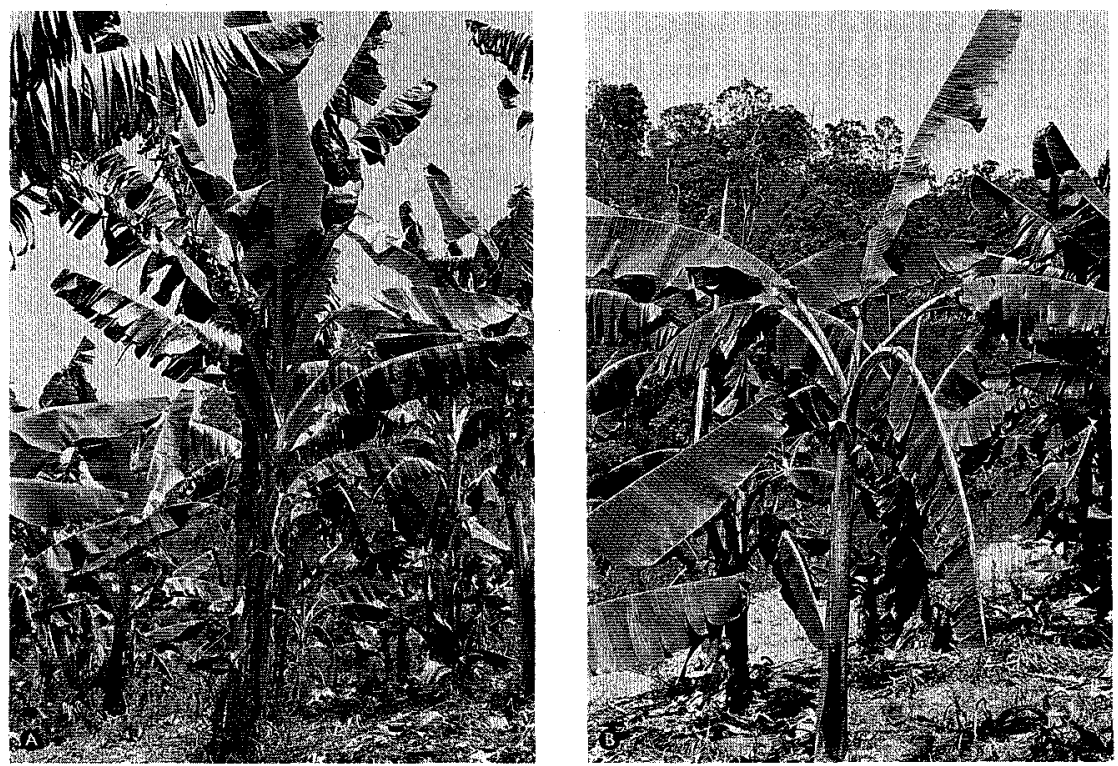

Fig. 5. Micropropagated banana plants established in the field. A. diploid (SH-3362). B. autotetraploid (induced by colchicine treatment of micropropagated banana diploid SH-3362) which exhibits weak petioles and drooping leaves, a feature of tetraploids.

Our work has shown that high percentages of autotetraploid bananas can be induced following exposure to $0.5 \%$ colchicine for $2 \mathrm{~h}$, with or without the use of $2 \%$ DMSO (Fig. 4). Of the total shoot tips treated, over $30 \%$ were induced to the tetraploid level. DMSO increased the mortality of plantlets when used with higher concentrations of colchicine; however, of the plants that survived a high percentage were tetraploids $(>50 \%)$. This is similar to the observation of Omar et al. (1989) who used DMSO to improve the uptake of ethylmethane sulfonate into banana shoot tips for mutation induction.

Vakili (1962) also found $0.5 \%$ colchicine applied to banana seeds for $2 \mathrm{~h}$ was the best treatment for tetraploid induction. Unfortunately Vakili was using seed and the performance and disease resistances of the resultant plants were unknown. The advantage of using micropropagated bananas is that a large number of plants can be produced for colchicine induction and these plants are well characterised. By using improved diploids from established breeding programs there is a greater chance of producing tetraploids with the necessary genes to confer disease resistance or improved yield. By using this material in a breeding program with selected diploids, the chances of obtaining superior triploid progeny are increased. The autotetraploids produced here from the race 4 Fusarium wilt-resistant diploid, SH-3362, have been returned to FHIA for inclusion in their breeding program.

The autotetraploids produced from this study have exceptionally weak petioles and produce few suckers (Fig. 5). The cold susceptibility of SH-3362 also appears to be exaggerated in the autotetraploid. However, the resistance of the diploid to Fusarium wilt is also shown by the autotetraploid and this has implications for breeding triploid progeny with resistance to race 4 .

Stable autotetraploids have been established in the field, but reversions and formation of chimeras and of aneuploids were common. Reversion to the diploid level was verified with stomatal measurements of leaves and chromosome counts from root-tip squashes. Reversion was encountered during micropropagation of colchicine-treated plants and after field 
establishment. Again the main advantage of micropropagation is that large numbers of plants can be produced and, even allowing for unstable lines, a sufficient number of stable clones can be generated for field evaluation and for inclusion into breeding programs.

This work on autotetraploid production originated from the need to introduce genetic diversity into breeding programs in an effort to develop clones resistant to diseases such as Fusarium wilt and black Sigatoka (Stover and Buddenhagen 1986). The technique reported here produces autotetraploids quickly and simply and should find application in breeding programs or in situations where cultivated diploids can be induced to a tetraploid level in order to improve fruit size. At present micropropagation is the most reliable method for producing large numbers of plants for colchicine induction, and can be used to great advantage for the induction of high percentages of autotetraploids in banana improvement programs.

\section{Acknowledgments}

We thank R. Drew for critically reviewing the manuscript. This work was supported by the Australian Centre for International Agricultural Research (ACIAR).

\section{References}

Blakeslee, A.F., and Avery, A.G. (1937). Methods of inducing doubling of chromosomes in plants by treatment with colchicine. Journal of Heredity 28, 393-411.

Borges, F. (1971). Tamano y densidad de estomas en clones cultivados y especies silvestres de Musa. Agronomica Tropical (Venezuela) 21, 139-43.

Brake, V.M., Pegg, K.G., Irwin, J.A.G., and Langdon, P.W. (1990). Vegetative compatibility groups within Australian populations of Fusarium oxysporum f.sp. cubense, the cause of fusarium wilt of bananas. Australian Journat of Agricultural Research 41, 863-70.

Burnham, C. (1962). 'Discussions in Cytogenetics.' (Burgess Publishing Company: Minneapolis.)

Daniells, J., and Smith, M. (1991). Post-flask management of tissue cultured bananas. ACIAR Technical Report No 18. (Australian Centre for International Agricultural Research: Canberra.)

Dermen, H. (1940). Colchicine polyploidy and technique. The Botanical Review 6, 599-635.

Iyer, C.D.A., and Randhawar, G.S. (1965). Increasing colchicine effectiveness in woody plants with special reference to fruit crops. Euphytica 14, 293-5.

Krythe, J.M., and Wellensieke, S.J. (1952). Five years of colchicine research. Bibliographia Genetica $14,1-132$.

Martens, M.R., and Reisch, B.I. (1988). An improved technique for counting chromosomes in grapes. HortScience 23, 896-99.

Murashige, T., and Skoog, F. (1962). A revised medium for rapid growth and bioassays with tobacco tissue cultures. Physiologia Plantarum 15, 473-97.

Omar, M.S., Novak, F.J., and Brunner, H. (1989). In vitro action of ethylmethane sulphonate on banana shoot tips. Scientia Horticulturae 40, 283-95.

Pegg, K.G., and Langdon, P.W. (1987). Fusarium wilt (Panama Disease): a review. In 'Banana and Plantain Breeding Strategies'. Proceedings of an international workshop held at Cairns, Australia, 13-17 October, 1986. (Eds G.J. Persley and E.A. De Langhe.) pp. 119-123. (ACIAR Proceedings No. 21: Canberra.)

Persley, G.J., and De Langhe, E.A. (Eds) (1987). Banana and Plantain Breeding Strategies: Proceedings of an International Workshop held at Cairns, Australia, 13-17 October 1986. (ACIAR Proceedings No. 21: Canberra.)

Rowe, P.R. (1984). Breeding, bananas and plantains. In 'Plant Breeding Reviews'. (Ed. J. Janick.) Vol. 2, pp. 135-55. (AVI Publishing Company: Westport, Conn.)

Rowe, P.R., and Richardson, D.L. (1975). Breeding bananas for disease resistance, fruit quality and yield. Bulletin No. 2. December 1975. Tropical Agriculture Research Services. (SIATS: La Lima, Honduras.)

Sanford, J.C. (1983). Ploidy manipulations. In 'Methods in Fruit Breeding'. (Eds J.N. Moore and J. Janick.) pp. 100-23. (Purdue University Press: Indiana.)

Shepherd, K. (1987). Banana breeding, past and present. Acta Horticulturae 196, 37-43.

Simmonds, N.W. (1966). 'Bananas.' 2nd Edition. (Longmans: London.) 
Smith, M.K., and Drew, R.A. (1990). Current applications of tissue culture in plant propagation and improvement. Australian Journal of Plant Physiology 17, 267-89.

Stover, R.H., and Buddenhagen, I.W., (1986). Banana breeding, polyploidy, disease resistance and productivity. Fruits 41, 175-91.

Stover, R.H., and Simmonds, N.W. (1987). 'Bananas.' 3rd Edition. (Longmans: London.)

Vakili, N.G. (1962). Colchicine-induced polyploidy in Musa. Nature 194, 453-4.

Vakili, N.G. (1967). The experimental formation of polyploidy and its effect in the genus Musa. American Journal of Botany 54, 24-36.

Manuscript received 5 June 1992, accepted 30 September 1992 